\title{
Hepatocyte growth factor and HER2/neu downregulate expression of apoptosis-inducing factor in non-small cell lung cancer
}

\author{
YUNG-YEN CHIANG $^{1}$, KUAN-CHIH CHOW ${ }^{2}$, TZE-YI LIN ${ }^{3}$, I-PING CHIANG ${ }^{3}$ and HSING-YUAN FANG ${ }^{4}$ \\ ${ }^{1}$ Department of Dental Laboratory Technology, Central Taiwan University of Science and Technology; \\ ${ }^{2}$ Graduate Institute of Biomedical Sciences, National Chung Hsing University; Departments of \\ ${ }^{3}$ Pathology and ${ }^{4}$ Surgery, China Medical University Hospital, Taichung, Taiwan, R.O.C.
}

Received August 9, 2013; Accepted September 27, 2013

DOI: $10.3892 /$ or.2013.2867

\begin{abstract}
Our previous study showed that patients with advanced stages of non-small cell lung cancer (NSCLC) were frequently detected with upregulation of hepatocyte growth factor (HGF). In vitro, HGF reduced expression of apoptosis-inducing factor (AIF) and cisplatin sensitivity in NSCLC cells. The effect of HGF was via HGF receptor (c-MET) and the downstream effector, focal adhesion kinase (FAK). In this study, we determined the prognostic value of AIF in NSCLC patients. AIF expression was determined by immunohistochemistry and immunoblotting. Our data show that AIF expression was associated with better prognosis. Expression of AIF inversely correlated with that of positive NSCLC markers, e.g., dihydrodiol dehydrogenase (DDH), c-MET, short oncostatin M receptor (OSMRs), matrix metalloproteinase (MMP)-1, and HER2/neu, which were closely associated with drug resistance, tumor recurrence, metastasis and poor prognosis. Noteworthy, silence of HER2/neu gene expression increases AIF level and drug sensitivity. Addition of HGF inhibits AIF expression in HER2/neu-silenced cells. These results suggested that both HGF and HER2/neu affect drug resistance by regulating AIF expression in NSCLC.
\end{abstract}

Correspondence to: Professor Kuan-Chih Chow, Graduate Institute of Biomedical Sciences, National Chung Hsing University, 250 Kuo-Kuang Road, Taichung 40227, Taiwan, R.O.C.

E-mail: kcchow@dragon.nchu.edu.tw

Abbreviations: AIF, apoptosis inducing factor; ATII, alveolar type II epithelial cells; ATM, ataxia-telangiectasia mutated kinase; ATR, ATM and Rad 3-related kinase; HGF, hepatocyte growth factor; HGFR, HGF receptor/ c-MET; MMP-1, matrix metalloproteinase-1; NSCLC, non-small cell lung cancer; PI3K, phosphoinositide 3-kinase

Key words: apoptosis inducing factor, cigarette smoking, cisplatin resistance, hepatocyte growth factor, non-small cell lung cancer

\section{Introduction}

Lung cancer is one of the leading causes of cancer death worldwide. In the United States, the annual mortality rate of lung cancer (estimated 180,000 deaths, male: $73.5 / 10^{5}$ personyear; female: $41.5 / 10^{5}$ person-year) is approximately $30 \%$ of total cancer-related deaths (1), and nearly $85-90 \%$ of lung cancer deaths are attributed to tobacco smoking (2). In Taiwan, the annual mortality rate of lung cancer is approximately $20 \%$ (estimated 6,000 deaths, male: $21 / 10^{5}$ person-year; female: $10.3 / 10^{5}$ person-year) (3). Lung carcinoma is categorized into small cell lung cancer (SCLC) or non-small cell lung cancer (NSCLC) with neuroendocrine features of the cancer cells. Based on the histopathological characteristics, NSCLC can be subcategorized into adenocarcinoma (ADC), squamous cell carcinoma and large cell carcinoma (4). Of note, among these, ADC, which is associated with a higher frequency of drug resistance and mortality than the other types, is most commonly found in women and smokers (5).

Previous studies have indicated that tobacco smoking is a key risk factor for lung cancer $(1,2,5,6)$. In patients with stage I NSCLC, we demonstrated that tobacco smoking and tumor size, but not visceral pleural invasion, are major factors influencing overall and disease-free survival (6). Moreover, accumulated evidence showed that NSCLC patients who continued to smoke were more resistant to chemotherapy and irradiation, and had poorer prognosis (6-9). Although nicotine per se is not directly associated with tumorigenesis, catalyzed nicotine is carcinogenic $(10,11)$. In addition, nicotine induces NSCLC growth, and increases angiogenesis in tumors probably via activating nicotinic acetylcholine receptor (nAchR), epidermal growth factor receptor (EGFR) and Akt (12-19).

Using differential display alone or in combination with microarray, we previously identified a spectrum of NSCLCspecific tumor markers, such as dihydrodiol dehydrogenase (DDH), c-MET, matrix metalloproteinase (MMP) and short oncostatin M receptor (OSMRs), which were closely associated with resistance to chemotherapy, tumor recurrence, metastasis and poor prognosis (20-24). Although we expected that DDH would correlate with drug resistance (20-22) and MMP with tumor metastasis (23), DDH was not directly involved in 
cisplatin deactivation. However, since DDH overexpression is closely related to tobacco smoking, and tobacco smoking is the key risk factor for carcinogenesis and disease progression of lung cancer, we used the same methods to examine gene expression profiles in biopsy specimens from smokers and nonsmokers, and we detected that hepatocyte growth factor (HGF) was frequently overexpressed in smokers with NSCLC. HGF was correlated with tumor stages and poor prognosis (25). Of note, HGF not only increased resistance to cisplatin, but also reduced levels of apoptosis inducing factor (AIF), a vital factor of caspase-independent cell death (CICD), in $\operatorname{NSCLC~}(25,26)$. Reduced effect of EGFR tyrosine kinase inhibitor (TKI), gefitinib, in patients with smoking habits or amplified c-MET gene suggested that in addition to cisplatin HGF might be involved in resistance to TKI as well (27). However, the prognostic value of HGF downstream effector AIF and its association with metastasis-related genes in NSCLC have not been reported.

In this study, we investigated AIF expression, and evaluated the statistical relationship between AIF expression and the clinicopathological factors as well as the prognostic significance of AIF expression in patients with NSCLC. We also studied the biological correlation between AIF and positive tumor marker genes in NSCLC.

\section{Materials and methods}

Tissue specimens. From August 1986 to November 2003, pathology specimens from 452 patients with NSCLC were reviewed. Pathology samples from all patients, for whom at least one follow-up examination or death was documented, were pathologically confirmed NSCLC. Of the 452 patients, 219 were diagnosed as having lung ADC. The stage of the disease was classified (patients after 1999) or re-classified (patients before 1999) according to the new international staging system for lung cancer (28). The Medical Ethics Committee approved the protocol in 2001, and written informed consent of donating biopsy specimens had been obtained from every patient before surgery since 2001. All patients had undergone surgical resection and radical N2 lymph node dissection. Tumor size, lymph node number, differentiation, vascular invasion and mitotic number were documented. Patients with lymph node involvement or locoregional recurrence received irradiation at the afflicted areas. Those with distant metastasis were treated with chemotherapy. After treatment, patients were routinely followed every 3 to 6 months in the outpatients department. Tumor recurrence and metastasis were diagnosed when biochemical studies, chest radiography, whole body bone scan and computerized tomography scans of chest showed any evidence of the disease. Immunohistochemical staining was carried out using a single-blinded procedure.

Immunoblotting analysis. Total cell lysate was prepared by mixing $5 \times 10^{7}$ cells $/ 100 \mu \mathrm{l}$ phosphate-buffered saline with equal volume of $2 \mathrm{X}$ loading buffer $(50 \mathrm{mM}$ Tris, $\mathrm{pH} 6.8$, $150 \mathrm{mM} \mathrm{NaCl}, 1 \mathrm{mM}$ disodium EDTA, $1 \mathrm{mM}$ PMSF, $10 \%$ glycerol, $5 \% \beta$-mercaptoethanol, $0.01 \%$ bromophenol blue and $1 \%$ SDS). Eletrophoresis was carried out in two $10 \%$ polyacrylamide gels with $4.5 \%$ stacking. One gel was processed for immunoblotting (20-26), and the other gel was stained with Coomassie blue. After electrophoresis, proteins on the first gel were transferred to a nitrocellulose membrane for immunoblotting. The membrane was probed with specific antibodies. The signal was amplified by biotin-labeled goat anti-mouse $\mathrm{IgG}$, and peroxidase-conjugated streptavidin. The protein was visualized by exposing the membrane to an X-Omat film (Eastman Kodak, Rochester, NY, USA) with enhanced chemiluminescent reagent (NEN, Boston, MA, USA). After getting unsatisfactory results from several batches of commercially obtained antibodies for Her2/neu, we decided to raise our own antibodies.

Preparation of mouse antibodies to HER2/neu. DNA sequence corresponding to C-terminal amino acids 807-1183 of HER2/neu was amplified by primer sequences containing SalI (sense) and NotI (antisense) restriction sites respectively. The primer sequences were 5'-TCCGTCGACAAATGGACCAT GTCCGGGAAAAC-3' (SalI site is underlined) and 5'-AGC GGCCGCAGTCTTTGACGACCCCATTCTT-3' (NotI site is underlined).

The 1131-bp cDNA of HER2/neu was cloned into an expression vector pET-32a $\mathrm{a}^{+}$(Promega KK, Tokyo, Japan). Bacterial colony containing the pET32+-HER $2 /$ neu was selected, and induced by isopropyl- $\beta$-D-thiogalactopyranoside (IPTG) to mass-produce HER2/neu. The recombinant protein was purified by a nickel-affinity column, and protein identity was determined by MALDI-TOF. Affinity-purified HER2/neu was used to immunize BALB/c mice, and sensitivity of antiserum $\left(\mathrm{OD}_{405}>0.3\right.$ at 1:6,000 dilutions) was measured by enzyme-linked immunosorbent assay (ELISA). Specificity of antibodies was determined by showing distinct bands with molecular weight of $185 \mathrm{kDa}$ in the immunoblotting of breast cancer cell extract. Monoclonal antibodies were produced by a hybridoma technique, and HER2/neu-specific antibodies were screened by the above-mentioned methods.

Immunohistochemistry. Immunohistochemical staining was performed according to the immunoperoxidase method previously reported (20-26).

Slide evaluation. In each pathological section, non-tumor lung tissue (NTLT) served as the internal negative control. Slides were evaluated by two independent pathologists blinded to the clinicopathological knowledge. The ImmunoReactive Scoring system was adapted for this study (29). Briefly, a specimen was considered having strong signals when $>50 \%$ of cancer cells were positively stained; intermediate, if $25-50 \%$ of the cells stained positive; weak, if $<25 \%$ or $>10 \%$ of the cells were positively stained; and negative, if $<10 \%$ of the cells were positively stained. Cases with strong and intermediate AIF signals were classified as $\mathrm{AIF}^{+}$, and those with weak or negative AIF signals were classified as AIF-. Those with AIF detecting in the nuclei were classified as cases with nuclear AIF index (NAI).

Statistical analysis. The relationship between AIF expression and clinicopathological parameters was analyzed by Chi-square test. Survival curves were plotted using the KaplanMeier estimator (30). Statistical difference in survival among different groups was compared by the log-rank test (between $\mathrm{AIF}^{+}$and $\mathrm{AIF}^{-}$groups) and log-rank test for trend [among NAI, 

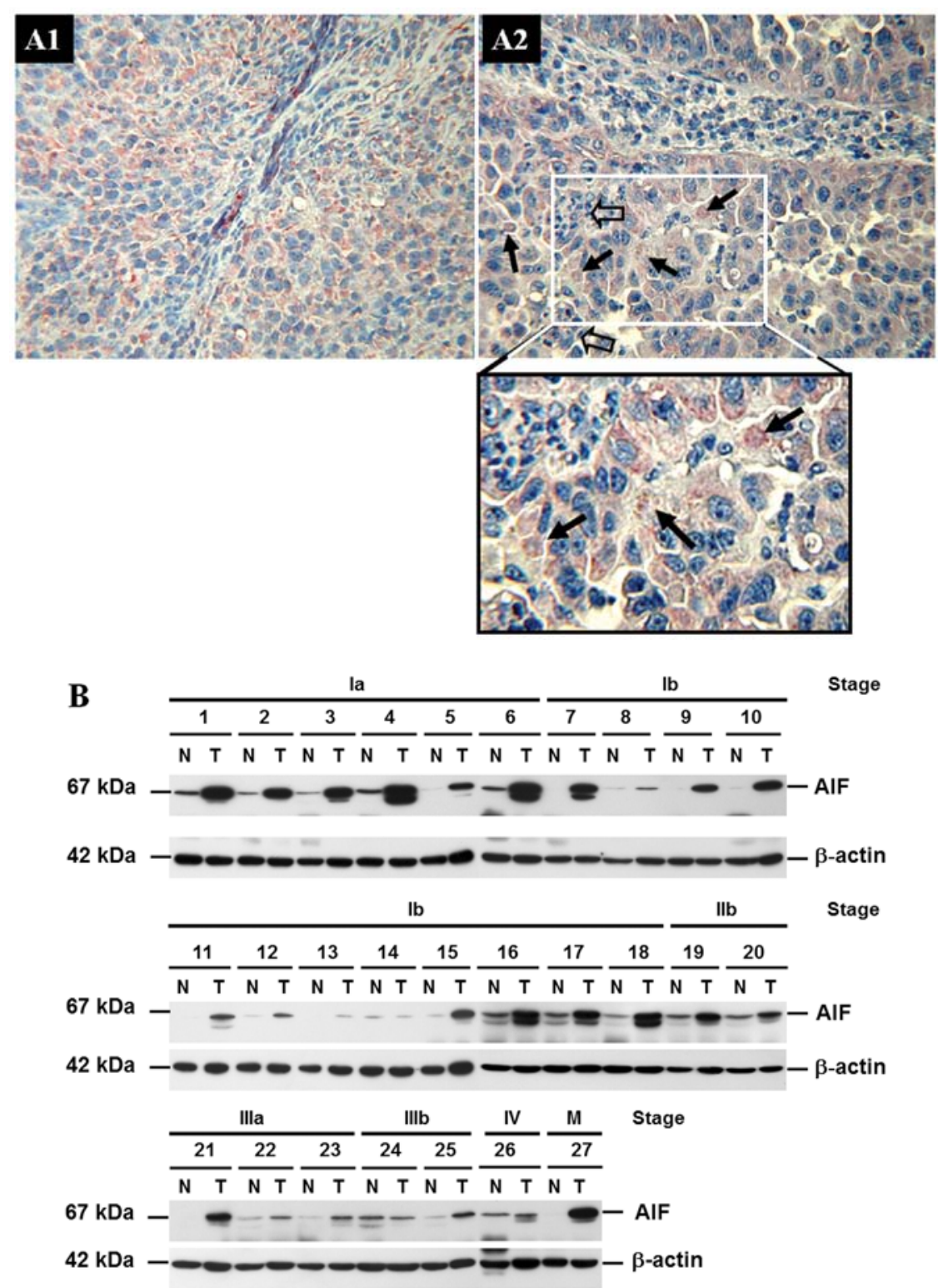

Figure 1. Expression of AIF in pathological specimens of NSCLC. (A) Representative examples of AIF expression in NSCLC. (A1) Expression of AIF was detected by immunohistochemistry (as crimson color precipitates in cytoplasm). (A2) Some AIF signals were detected in nuclei of tumor cells (indicated by arrows), when infiltrates of white blood cells were identified around tumor nests (indicated by white arrows). (B) Expression of AIF was confirmed by immunoblotting. N, non-tumor lung tissue; $\mathrm{T}$, tumor fraction of surgical resections.

$\mathrm{cAIF}^{+}$(cytoplasmic AIF, AIF ${ }^{+}$patients minus NAI patients) and AIF- groups] (31). Statistical analysis was performed using GraphPad Prism5 statistical software (San Diego, CA, USA). Statistical significance was set at $\mathrm{p}$-value $<0.05$.

\section{Results}

Expression of AIF in NSCLC and correlation with patient survival. Using AIF-specific monoclonal antibodies, we detected AIF expression (Fig. 1A1 and A2) in tumor cells in 109 NSCLC patients (24.1\%). In 14 (12.84\%) of the 109 patients, some AIF signals were identified in the nuclei of tumor cells (Fig. 1A2). Nuclear AIF indicated that these tumor cells are under apoptosis. Judging from vicinity of the tumor, apoptotic cancer cells could be associated with the infiltrating immune cells. AIF was also detected in $40.45 \%$ (36/89) of metastatic lymph nodes. However, no nuclear AIF was identified in the metastatic lymph nodes. AIF expression in was verified by immunoblotting (Fig. 1B). Expression of AIF decreased following advances of tumor stage. From stage 1b, AIF level reduced markedly.

Among the 109 patients whose tissue samples had high AIF expression, 31 (28.44\%) patients had tumor recurrence. Among the 343 patients whose tissue samples had low AIF expression, 168 (48.98\%) patients had tumor recurrence during follow-up examination. All 199 patients who had recurrence developed new tumors within 18 months after operation and cisplatin-based chemotherapy. Recurrence rate of patients with low AIF expression was 1.72-fold higher than that of patients with high AIF expression. The difference was significant $(p<0.01)$. Moreover, survival of patients with high AIF levels was significantly better than that of patients with low AIF 
A

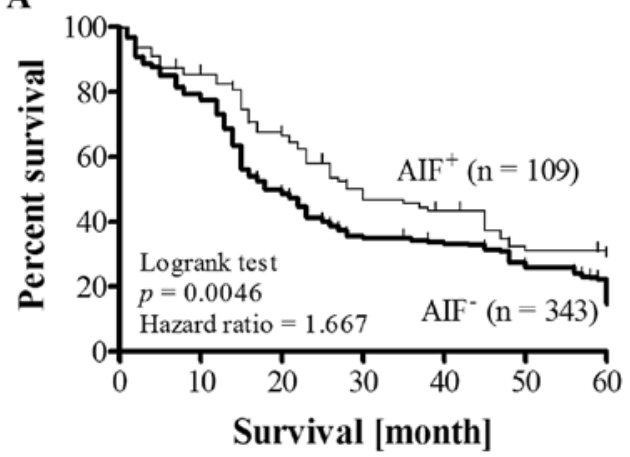

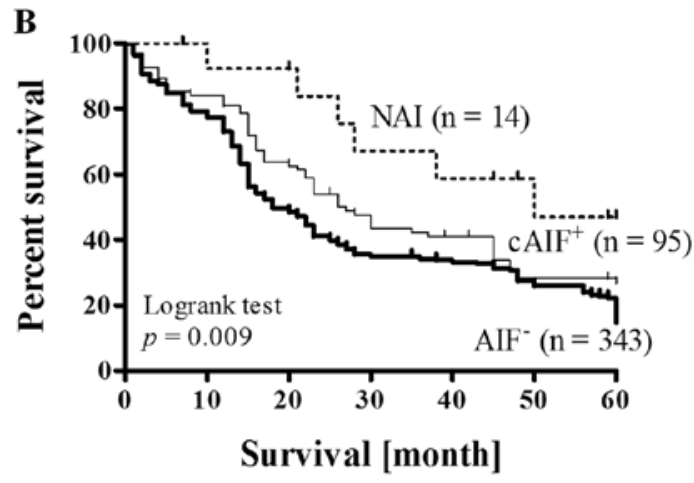

Figure 2. Comparison of Kaplan-Meier product limit estimates of survival analysis in patients with NSCLC. (A) Patients were divided into two groups based on AIF expression in pathological specimens of NSCLC. AIF-, NSCLC with low AIF expression; AIF $^{+}$, NSCLC with high AIF expression. Survival difference between the two groups was compared by a log-rank test. $\mathrm{p}=0.0046$; (B) Patients were divided into three groups based on AIF expression and detection of AIF in the nuclei of NSCLC. Survival rate in patients with nuclear AIF index (NAI) was the best among three patient groups. The difference in cumulative survival among the three groups was significant $(\mathrm{p}=0.009)$.
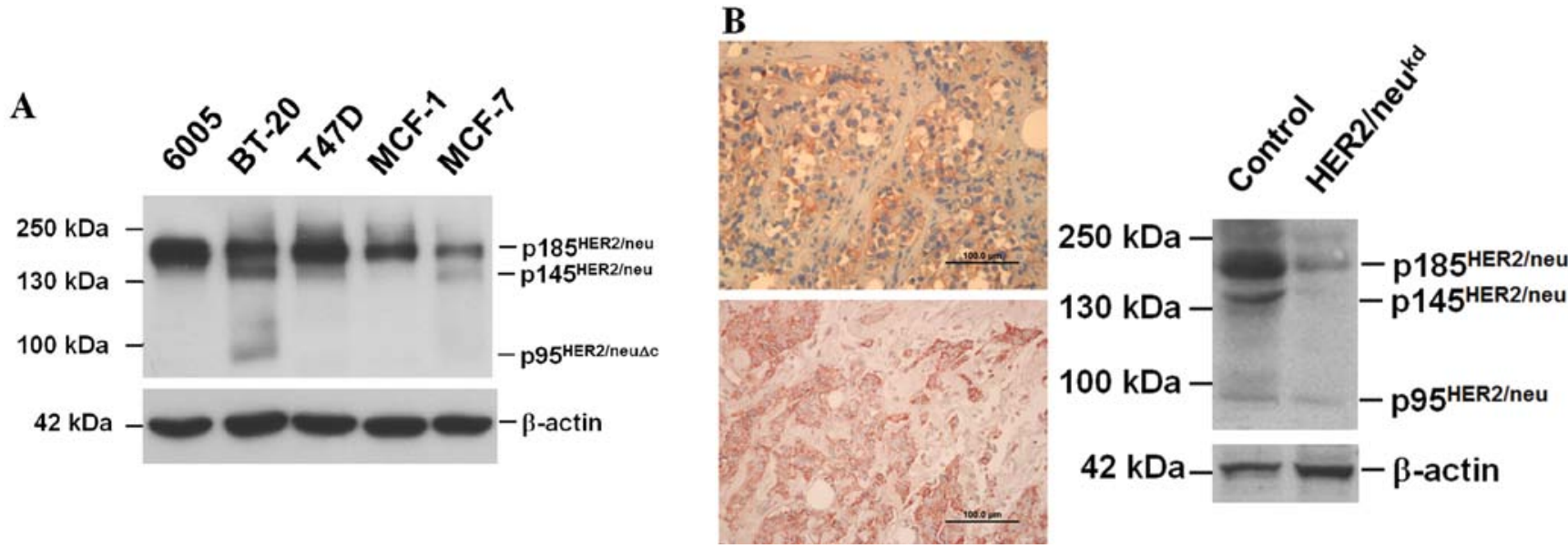

Figure 3. Characterization of monoclonal antibodies to HER2/neu. (A) A unique 185-kDa protein (p185 ${ }^{\text {HER } 2 / n e u}$ ) was detected by immunoblotting in breast cancer cells. In some cancer cell lines, p145 ${ }^{\mathrm{HER} 2 / \text { neu }}$ and $\mathrm{p} 95^{\mathrm{HER} 2 / \mathrm{neu} \Delta \mathrm{c}}$ (HER2/neu with deletion of extracellular domain) fragments were detected. (B) Immunohistochemical staining showed that HER2/neu was abundantly present on the plasma membranes of breast cancer cells (left panels). Infection with lentivirus expressing siRNA to knockdown HER2/neu (HER2/neu ${ }^{\mathrm{kd}}$ ) expression validated that our monoclonal antibodies uniquely recognized HER2/neu (right panel). Reduction of HER2/neu expression is $~ 87 \%$ as determined by band intensity scan (EDAS 290, Kodak, Rochester, NY, USA).

levels (Fig. 2A). Survival rate of patients with high nuclear AIF index (NAI) was the best among patient groups (Fig. 2B). The differences in cumulative survival between groups were significant $(\mathrm{p}=0.0046$ and $\mathrm{p}=0.009)$.

Functional characterization of monoclonal antibodies to HER2/neu. Specificity of the monoclonal antibodies was determined by an immunoblotting analysis of the whole cell lysate. The unique $185-\mathrm{kDa}$ protein was detected by monoclonal antibodies (Fig. 3A). Immunohistochemical staining showed that HER2/neu was present on plasma membranes of breast cancer cells (Fig. 3B, left panels). Infection with lentivirus expressing siRNA to HER2/neu reduced HER2/neu expression validated that our monoclonal antibodies uniquely recognized HER2/neu (Fig. 3B, right panel).

Levels of AIF inversely correlates with expression of positive tumor markers in NSCLC. Our previous studies showed that overexpression of DDH, c-MET, MMP1 and OSMRs in NSCLC was associated with drug resistance, tumor recur- rence, metastasis and poor prognosis (20-26). Moreover, our recent data suggested that expression of HGF, which was frequently detected in smokers with NSCLC, reduced levels of AIF (25). In this study, we investigated the correlation between levels of AIF and expression of positive tumor markers, e.g., DDH, c-MET, MMP1, HGF, OSMRs and HER2/neu (Fig. 4A), in NSCLC. HER2/neu in patients with the early stages was mainly p95 ${ }^{\mathrm{HER} 2 / \mathrm{neu} \Delta \mathrm{c}}$ (HER2/neu with deletion of extracellular domain) (32); in advanced stages it was the $185^{\mathrm{HER} 2 / \text { neu }}$. As shown in Fig. 4B, higher AIF expression ratio was detected in $37(43.5 \%)$ of 85 tumor specimens. When gene expression, tumor staging and smoking habit were used to categorize patient groups, our data showed that AIF expression, as determined by immunoblotting, was inversely correlated with positive tumor markers, tumor staging and cigarette smoking. Noteworthy, cancer samples that had higher level of HER2/neu expressed less AIF. Levels of checkpoint kinase 1 (CHK1) and Nijmegen breakage syndrome 1 (NBS1, nibrin) protein, which are essential for mediating cell cycle arrest and maintaining genome stability during DNA replication, on the other hand, 

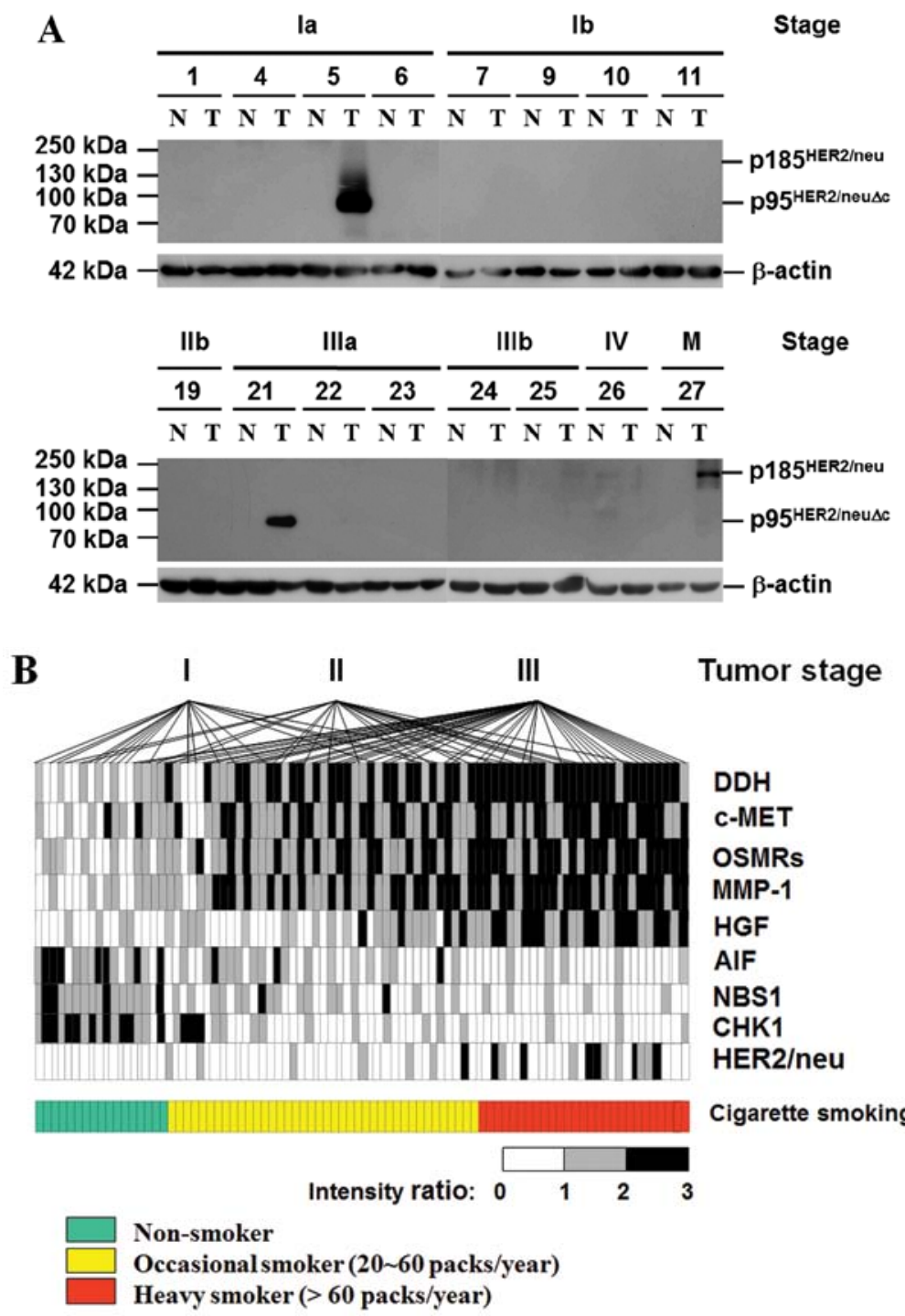

Figure 4. Expression of AIF and some positive tumor markers in NSCLC. (A) Expression of HER2/neu was detected by immunoblotting. Expression of $\beta$-actin was used as a monitoring standard. N, non-tumor lung tissue; T, tumor fraction of surgical resections. (B) Gene cluster analysis of 85 human NSCLC samples by AIF expression in patients with NSCLC. Level of gene expression was determined by measuring the intensity ratio between the specific protein and $\beta$-actin of immunoblots.

were proportional to those of AIF. The results are consistent with our in vitro data that AIF level is comparative to that of NSB1 (22) and HGF inhibits AIF expression (26).

In vivo, expression level of AIF is comparatively low in the center of cigarette smoking (CS)-induced lung adenocarcinoma in $B A L B / c 3$ mice. To study the in vivo effect of cigarette smoking on AIF expression, male BALB/c mice (National Animal Center, National Science Council, Taipei, Taiwan) at six weeks of age were treated with passive cigarette smoking daily ( 5 min a day in a $40 \mathrm{~cm} \mathrm{x} 40 \mathrm{~cm}$ x $60 \mathrm{~cm}$ chamber, and each cigarette contained $0.9 \mathrm{mg}$ of nicotine, Marlboro, USA) for 25 weeks before histopathological and biochemical examinations (Animal exposure protocols were approved by the Institutional Animal Care and Use Committee of National Chung-Hsing University). Among 100 male mice, lung cancer was detected in seven mice. Compared to alveolar type II (ATII) pneumocytes and cancer cells in the periphery (Fig. 5A), which were highly expressing AIF, AIF level decreased toward the center of the tumor (Fig. 5B). The results are consistent with our in vitro data that nicotine reduced AIF expression $(26,33)$.

Effect of HER2/neu on AIF expression and cell survival following cisplatin challenge in NSCLC cells. Eight NSCLC cells, H23, H225, H226, H838, H1437, H2009, H2087 and A549, examined by immunoblotting, expressed various levels of HER2/neu: high in H2009 and H2087; and low in H838 and A549 (Fig. 6A). HER2/neu was not detected in H23, H225, $\mathrm{H} 226$ and H1437. Of note, silence of HER2/neu expression by siRNA increased AIF expression; however, it did not affect the HGF effect on downregulation of AIF (Fig. 6B). The data indicated that HER2/neu also influences AIF expression in NSCLC cells. Since c-MET and HER2/neu share FAK as a common signal transducer, we examined the effect of p $60^{\text {src }}$ and $\mathrm{p} 23^{\text {ras }}$ on FAK expression. As shown in Fig. 6C, overexpression of $\mathrm{p} 60^{\mathrm{src}}$ protected FAK protein; however, p23 ${ }^{\text {ras }}$ had no effect. The presence of HGF saved FAK protein from proteolytic degradation and facilitated cell moving out of the agarose trap (Fig. 6D). 


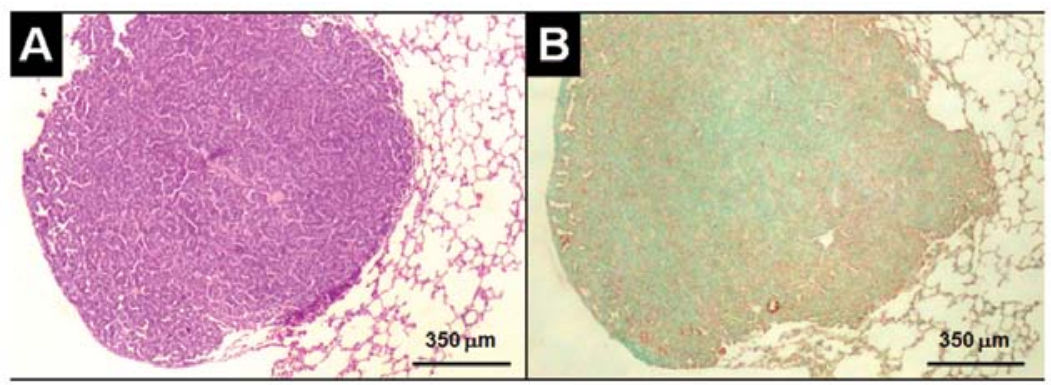

Figure 5. Expression level of AIF is comparatively low toward the center of cigarette smoking-induced lung adenocarcinoma in BALB/c3 mice. Male BALB/c mice (National Animal Center, National Science Council, Taipei, Taiwan) at six weeks of age were treated with passive cigarette smoking daily (5 min per day in a $40 \mathrm{~cm}$ x $40 \mathrm{~cm}$ x $60 \mathrm{~cm}$ chamber, and each cigarette contained $0.9 \mathrm{mg}$ of nicotine, Marlboro, USA) for 25 weeks before histopathological and biochemical examinations. Among 100 male mice, lung cancer was detected in seven mice. (A) Representative example of cigarette smoking-induced lung adenocarcinoma in BALB/c3 mice (hematoxylin-eosin-stained); (B) Compared to ATII pneumocytes and cancer cells in the periphery, which highly expressed AIF, AIF level decreased toward the center of tumor. Expression of AIF was detected by immunohistochemistry (as crimson color precipitates in cytoplasm). The slide was counterstained with methyl green.
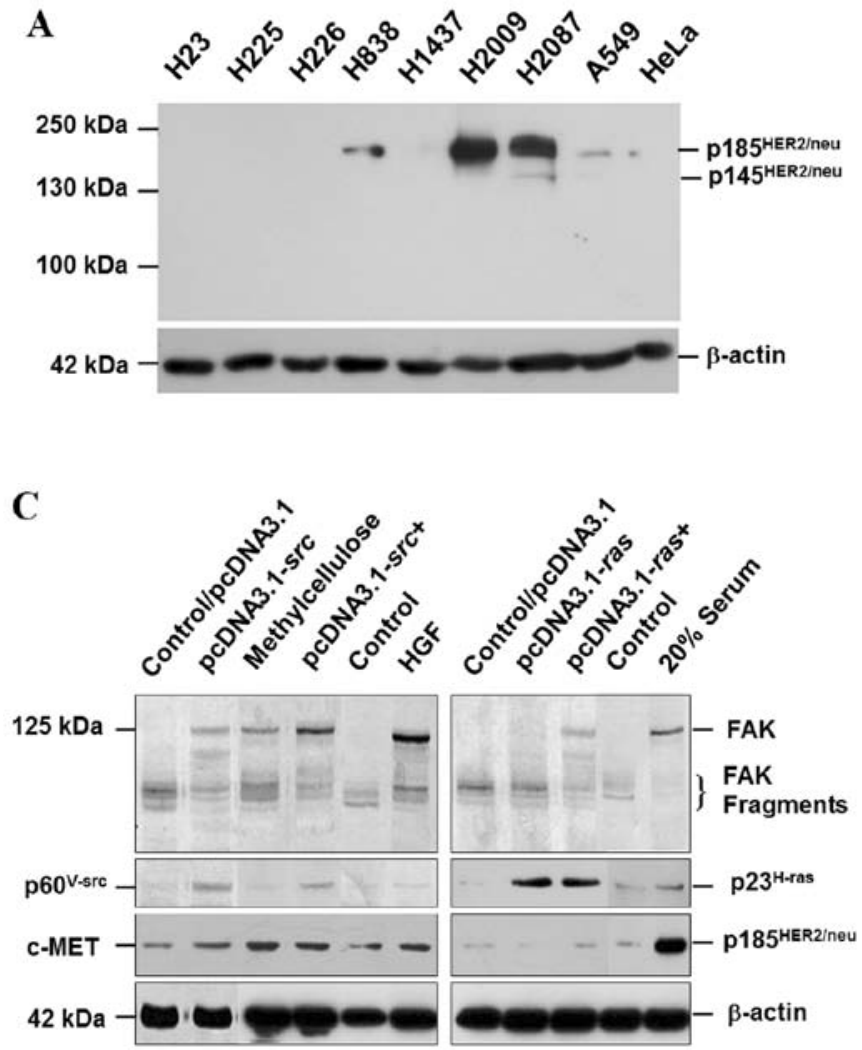

B
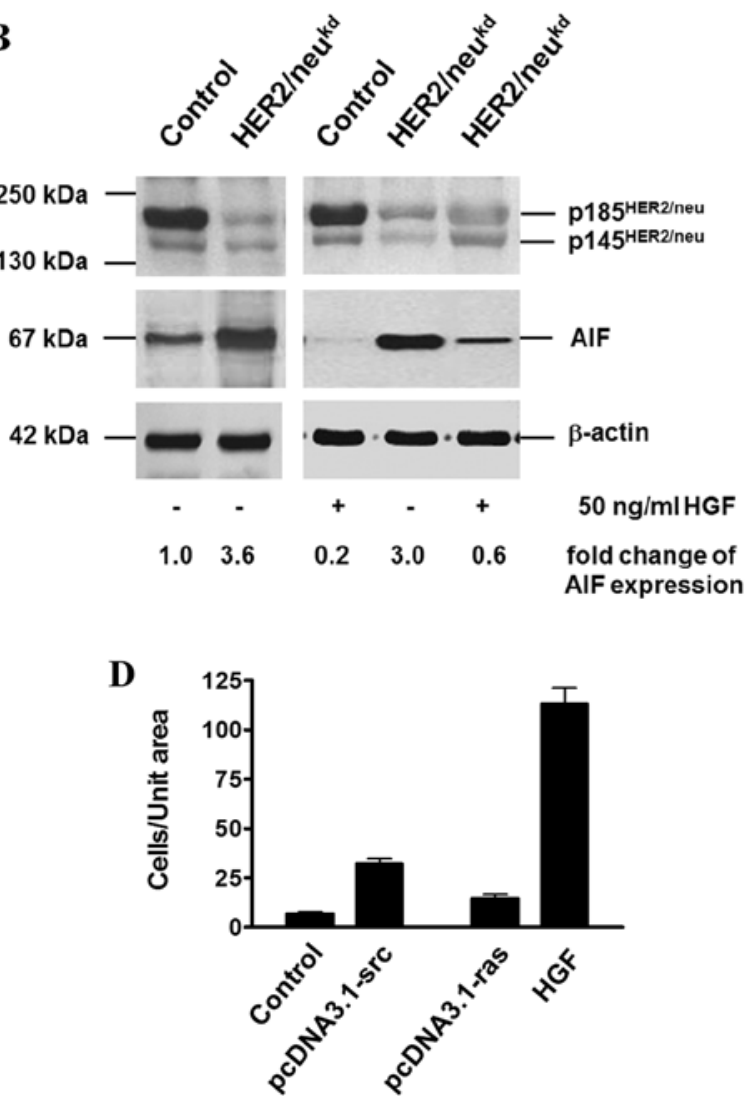

Figure 6. Expression of HER2/neu and the influence of HER2/neu on AIF expression in cultured ADC cells. (A) Expression of HER2/neu was detected in four human lung ADC cell types by immunoblotting. Expression of HER2/neu was high in H2009 and H2087, and low in H838 and A549. HER2/neu was not detected in H23, H225, H226 and H1437. (B) Infection with lentivirus expressing siRNA to knock down HER2/neu (HER2/neu ${ }^{\mathrm{kd}}$ ) expression increases AIF levels. Addition of HGF enhances the inhibitory effect of HER2/neu on AIF expression. (C) Transfection with pcDNA3.1-src carrying the full length src, or growing $\mathrm{H} 2126$ cells in $1 \%$ methylcellulose or addition of HGF increased expression levels of p125FAK. Expression of p125FAK was additively increased in pcDNA3.1-src-transfected H2126 cells which were grown in $1 \%$ methylcellulose. Although transfection with pcDNA3.1- $H$-ras did not affect p125FAK expression, growing pcDNA3.1- $\mathrm{H}$-ras-transfected $\mathrm{H} 2026$ cells in $1 \%$ methylcellulose increased expression levels of p125FAK. Addition of $20 \%$ fetal calf serum for $24 \mathrm{~h}$ increased both levels of HER2/neu and p125FAK. (D) The respective effect of p60 $0^{\mathrm{v}-\mathrm{src}}$ and $\mathrm{p} 23^{\mathrm{H}-\mathrm{ras}}$ on cell migration activity of H838 cells $\left(10^{5}\right.$ cells seeded in $0.3 \%$ agarose for $24 \mathrm{~h}$ ). Cells treated with $50 \mathrm{ng} / \mathrm{ml} \mathrm{HGF}$ were used as positive control.

\section{Discussion}

The results show that AIF expression in NSCLC is inversely correlated with tumor stage and patient cigarette smoking history. By demonstrating that in patients with more advanced lung cancer, especially, in those who smoked more than 20 pack-years, AIF expression was low in tumor cells $(\sim 67.6 \%$ of specimens), our data suggest that, besides being involved in lung carcinogenesis, cigarette smoking increases drug resistance by downregulating expression of AIF in lung tumor 


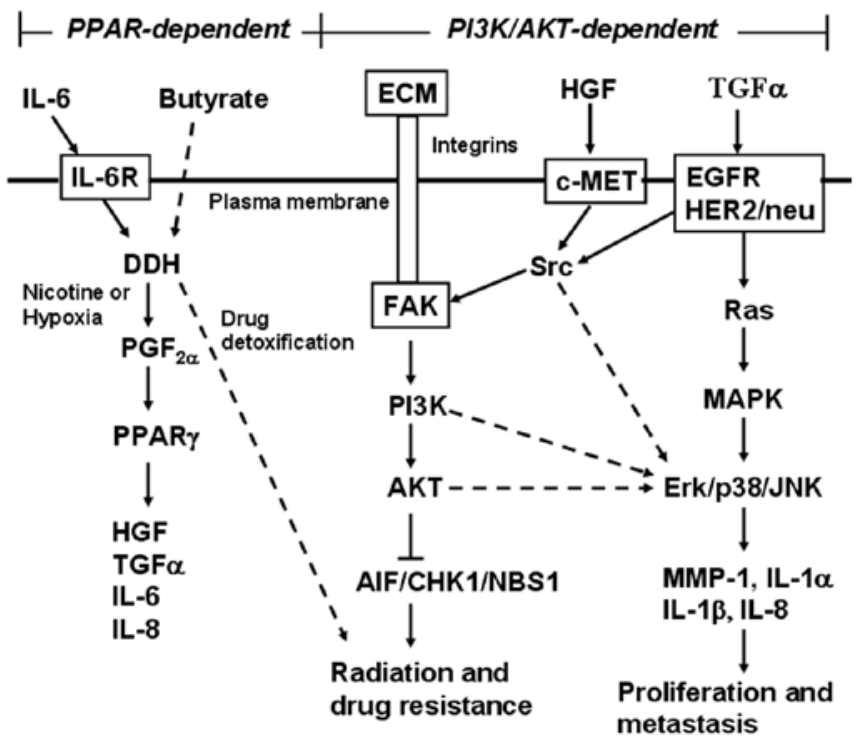

Figure 7. The putative network among integrins and receptor kinase-associated gene activation on expression of AIF, NBS1 and CHK1 as well as their consequences of drug- and radio-resistance in NSCLC.

cells. Reduced AIF expression correlated with poor prognosis and was inversely associated with expressions of positive tumor marker genes.

The results corresponded well with our previous study that HGF was upregulated by tobacco smoke in ATII and NSCLC cells (25). Increase of HGF in turn reduces AIF expression and cisplatin sensitivity (26), which could be mediated via c-MET, FAK, phosphoinositide kinase 3 (PI3K) and protein kinase B (PKB, also called AKT) pathway in NSCLC cells $(26,34)$. This study showed that HER2/neu was also involved in downregulation of AIF expression. Since signals from HER2/neu and c-MET converge onto a common transducer FAK protein, our results suggested that these two receptors might in part play a role in activation of PI3K and AKT (26), and activated AKT decreased expression levels of AIF, a vital factor of caspase-independent cell death, to induce drug resistance in NSCLC $(25,26)$. Expression level of FAK alone, however, was not associated with that of AIF or drug resistance (data not shown).

In addition, AIF level correlated with expression of critical sensor proteins of DNA damage and replication stress, NBS1 and $\mathrm{CHK} 1$, which are essential for mediating cell-cycle arrest and DNA-repair mechanisms (35). Noteworthy, FERM (band four point one, Ezrin/Radxin/Moesin) region of FAK protein interacts with the N-terminal transactivation domain of p53, which binds mouse double minute 2 (MDM-2), a ubiquitin ligase (36). Selective binding of p53 to MDM-2 or FAK facilitates cell transformation and epithelial-mesenchymal transition (37). By showing that overexpression of $\mathrm{v}$-src protein and addition of HGF or fetal calf serum (FCS) protected FAK from integrin-related protein degradation, our data suggested that integrin, v-src and H-ras might have different effects on FAK function. ATR, a cell stress-responsive kinase that is essential for sensing DNA replication error, contains a stretch of focal adhesion targeting (FAT) and PI3K motifs (38). Myers et al demonstrated that ATR and Chk1 alleviate replication-related stress by suppressing a caspase-3-dependent apoptotic response (39). Our data supported their findings and showed that in combination with reduced AIF expression, downregulation of NBS1 and CHK1, two mediators of ATR, might elude the inhibitory effect of p53 on cell cycle progression, and increase genomic heterogeneity as well as resistance to doxorubicin, etoposide, cisplatin and gemcitabine in NSCLC cells, which overexpressed HER2/neu (40). By showing that levels of HER2/neu increased following addition of FCS, our data suggested that HER2/neu expression might not be constitutive (41), but could be upregulated by yet to be determined serum factors.

Moreover, our previous studies showed that drug resistance, tumor recurrence, metastasis and poor prognosis of NSCLC correlated with overexpression of DDH, c-MET, MMP1, HGF and OSMRs (20-25). Recently, by confocal immunofluorescence microscopy we found that HGF, which was frequently detected in smoker patients, was expressed on surface of cancer cells (33). Therefore, the overexpressed HGF could only interact with nearby cells. Of note, HGF overexpression was induced by prostaglandin $\mathrm{F}_{2 \alpha}\left(\mathrm{PGF}_{2 \alpha}\right)$, which was synthesized by DDH when cells were under hypoxic condition (33). As shown in cigarette smoking-induced murine lung ADC, AIF expression was much lower in the center of tumor mass when tumor size was larger than one millimeter. However, we are less certain whether this phenomenon was caused by tumor size-related hypoxia or the effect of tobacco smoke could not reach the interior of tumor mass. In addition to reducing levels of AIF, HGF upregulates expression of interleukin (IL)- $1 \alpha,-1 \beta,-6,-8$ and -24 (a member of IL-10 family), as well as that of tumor necrosis factor (TNF) superfamily member 10 (TNFSF10), MMP1 and transforming growth factor $\alpha(\mathrm{TGF}-\alpha)$, an alternative ligand of EGFR (33). TGF- $\alpha$ activates EGFR and IL-6 induces overexpression of DDH (22), which constitute a vicious cycle to maintain cancer cell survival (Fig. 7); in particular, activated EGFR is vital for cell proliferation and DDH is essential for detoxification of anticancer drugs, including cisplatin (21), doxorubicin, etoposide, mitoxantrone, gefitinib and erlotinib, of which chemical structures are highly similar to polycyclic aromatic hydrocarbons (PAH) (20).

These results considered together with the current data provided in vitro explanations to support our previous findings that tobacco smoking and tumor size are the two major factors influencing overall and disease-free survival in patients with stage I NSCLC (6); in particular in patients with stage Ib disease AIF levels were markedly reduced. Patients who continued to smoke after proper resections were more resistant to chemotherapy and irradiation, and had poorer prognosis (6-8,20-25). Cigarette smoking and hypoxia respectively induce expression of $\mathrm{HGF}$, which decreases AIF levels and drug sensitivity in NSCLC cells $(25,26)$. In conclusion, our data show that AIF expression was frequently downregulated in patients with NSCLC, especially in those with a previous or current smoking history. Statistical analysis showed that decreased AIF level in NSCLC was closely associated with patient survival. These results suggest that cigarette smoking plays an important role in drug resistance and cancer cell survival, which were probably mediated via HGF, HER2/neu and FAK activation-induced downregulation of AIF, CHK1 and NBS1 expression in patients with NSCLC (42). 


\section{References}

1. Schiller JH, Harrington D, Belani CP, et al: The Eastern Cooperative Oncology Group. Comparison of four chemotherapy regimens for advanced non-small-cell lung cancer. N Engl J Med 346: 92-98, 2002.

2. Phillips DH: Smoking-related DNA and protein adducts in human tissues. Carcinogenesis 23: 1979-2004, 2002.

3. Annual reports of the Department of Health, the Executive Yuan, Taiwan, Republic of China, 2012.

4. Moran CA and Suster S: Tumours of the lung and pleura. In Diagnostic Histopathology of Tumours. Fletcher CDM (ed). Churchill Livingstone, London, pp171-208, 2000.

5. Ko YC, Lee CH, Chen MJ, et al: Risk factors for primary lung cancer among non-smoking women in Taiwan. Int J Epidemiol 26: 24-31, 1997

6. Hung JJ, Wang CY, Huang MH, Huang BS, Hsu WH and Wu YC: Prognostic factors in resected stage I non-small cell lung cancer with a diameter of $3 \mathrm{~cm}$ or less: visceral pleural invasion did not influence overall and disease-free survival. J Thorac Cardiovasc Surg 134: 638-643, 2007.

7. Browman GP, Wong G, Hodson I, et al: Influence of cigarette smoking on the efficacy of radiation therapy in head and neck cancer. N Engl J Med 328: 159-163, 1993.

8. Johnston-Early A, Cohen MH, Minna JD, et al: Smoking abstinence and small cell lung cancer survival. An association. JAMA 244: 2175-2179, 1980

9. Videtic GM, Stitt LW, Dar AR, et al: Continued cigarette smoking by patients receiving concurrent chemoradiotherapy for limited-stage small-cell lung cancer is associated with decreased survival. J Clin Oncol 21: 1544-1549, 2003.

10. Hoffmann D, Lavoie EJ and Hecht SS: Nicotine: a precursor for carcinogens. Cancer Lett 26: 67-75, 1985.

11. Minna JD: Nicotine exposure and bronchial epithelial cell nicotinic acetylcholine receptor expression in the pathogenesis of lung cancer. J Clin Invest 111: 31-33, 2003.

12. Garnier M, Lamacz M, Tonon MC and Vaudry H: Functional characterization of a nonclassical nicotine receptor associated with inositolphospholipid breakdown and mobilization of intracellular calcium pools. Proc Natl Acad Sci USA 91: 11743-11747, 1994.

13. Jull BA, Plummer HK III and Schuller HM: Nicotinic receptormediated activation by the tobacco-specific nitrosamine NNK of a Raf-1/MAP kinase pathway, resulting in phosphorylation of c-myc in human small cell lung carcinoma cells and pulmonary neuroendocrine cells. J Cancer Res Clin Oncol 127: 707-717, 2001.

14. Masaki T, Igarashi $\mathrm{K}$, Tokuda $\mathrm{M}$, et al: pp60c-src activation in lung adenocarcinoma. Eur J Cancer 39: 1447-1455, 2003.

15. Park PG, Merryman J, Orloff $M$ and Schuller HM: Betaadrenergic mitogenic signal transduction in peripheral lung adenocarcinoma: implications for individuals with preexisting chronic lung disease. Cancer Res 55: 3504-3508, 1995.

16. Schuller HM and Cekanova M: NNK-induced hamster lung adenocarcinomas over-express beta2-adrenergic and EGFR signaling pathways. Lung Cancer 49: 35-45, 2005.

17. Tsurutani J, Castillo SS, Brognard J, et al: Tobacco components stimulate Akt-dependent proliferation and NFkappaB-dependent survival in lung cancer cells. Carcinogenesis 26: 1182-1195, 2005.

18. West KA, Brognard J, Clark AS, et al: Rapid Akt activation by nicotine and a tobacco carcinogen modulates the phenotype of normal human airway epithelial cells. J Clin Invest 111: 81-90, 2003.

19. West KA, Linnoila IR, Belinsky SA, Harris CC and Dennis PA: Tobacco carcinogen-induced cellular transformation increases activation of the phosphatidylinositol 3'-kinase/Akt pathway in vitro and in vivo. Cancer Res 64: 446-451, 2004.

20. Hsu NY, Ho HC, Chow KC, et al: Overexpression of dihydrodio dehydrogenase as a prognostic marker of non-small cell lung cancer. Cancer Res 61: 2727-2731, 2001.
21. Deng HB, Parekh HK, Chow KC and Simpkins H: Increased expression of dihydrodiol dehydrogenase induces resistance to cisplatin in human ovarian carcinoma cells. J Biol Chem 277: 15035-15043, 2002.

22. Wang HW, Lin CP, Chiu JH, et al: Reversal of inflammationassociated dihydrodiol dehydrogenases (AKR1C1 and AKR1C2) overexpression and drug resistance in nonsmall cell lung cancer cells by wogonin and chrysin. Int J Cancer 120: 2019-2027, 2007.

23. Lin TS, Chiou SH, Wang LS, et al: Expression spectra of matrix metalloproteinases in metastatic non-small cell lung cancer. Oncol Rep 12: 717-723, 2004

24. Chen DR, Chu CY, Chen CY, et al: Expression of short form oncostatin $\mathrm{M}$ receptor as a decoy receptor in lung adenocarcinomas. J Pathol 215: 290-299, 2008.

25. Chen JT, Lin TS, Chow KC, et al: Cigarette smoking induces overexpression of HGF in type II pneumocytes and lung cancer cells. Am J Repir Cell Mol Biol 34: 264-273, 2006.

26. Chen JT, Huang CY, Chiang YY, et al: HGF increases cisplatin resistance via down-regulation of AIF in lung cancer cells. Am J Respir Cell Mol Biol 38: 559-565, 2008.

27. Yano S, Wang W, Li Q, et al: Hepatocyte growth factor induces gefitinib resistance of lung adenocarcinoma with epidermal growth factor receptor-activating mutations. Cancer Res 68: 9479-9487, 2008

28. Mountain CF: Revisions in the international system for staging lung cancer. Chest 111: 1710-1717, 1997.

29. Remmele W and Schicketanz KH: Immunohistochemical determination of estrogen and progesterone receptor content in human breast cancer. Computer-assisted image analysis (QIC score) vs subjective grading (IRS). Pathol Res Pract 189: 862-866, 1993.

30. Kaplan EL and Meier P: Nonparametric estimation from incomplete observations. J Am Stat Assoc 53: 457-481, 1958.

31. Mantel N: Evaluation of survival data and two new rank order statistics arising in its consideration. Cancer Chemother Rep 50: 163-170, 1966.

32. Swanton C, Futreal A and Eisen T: Her2-targeted therapies in non-small cell lung cancer. Clin Cancer Res 12: 4377s-4383s, 2006.

33. Chiang YY: Hepatocyte growth factor induces hypoxia-related interleukin-8 expression in lung adenocarcinoma cells. Mol Carcinog 48: 662-670, 2009.

34. Ma PC, Jagadeeswaran R, Jagadeesh S, et al: Functional expression and mutations of c-Met and its therapeutic inhibition with SU11274 and small interfering RNA in non-small cell lung cancer. Cancer Res 65: 1479-1488, 2005

35. Kastan MB and Bartek J: Cell-cycle checkpoints and cancer. Nature 432: 316-323, 2004

36. Lim ST, Chen XL, Lim Y, et al: Nuclear FAK promotes cell proliferation and survival through FERM-enhanced p53 degradation. Mol Cell 29: 9-22, 2008.

37. Mitra SK and Schlaepfer DD: Integrin-regulated FAK-Src signaling in normal and cancer cells. Curr Opin Cell Biol 18: 516-523, 2006.

38. Shiloh Y: ATM and related protein kinases: safeguarding genome integrity. Nat Rev Cancer 3: 155-168, 2003.

39. Myers K, Gagou ME, Zuazua-Villar P, Rodriguez R and Meuth M: ATR and Chk1 suppress a caspase-3-dependent apoptotic response following DNA replication stress. PLoS Genet 5: e1000324, 2009

40. Tsai CM, Chang KT, Wu LH, et al: Correlations between intrinsic chemoresistance and HER-2/neu gene expression, p53 gene mutations, and cell proliferation characteristics in non-small cell lung cancer cell lines. Cancer Res 56: 206-209, 1996.

41. Noro R, Gemma A, Kosaihira S, et al: Gefitinib (IRESSA) sensitive lung cancer cell lines show phosphorylation of Akt without ligand stimulation. BMC Cancer 6: 277, 2006.

42. King FW, Skeen J, Hay N and Shtivelman E: Inhibition of Chk1 by activated PKB/Akt. Cell Cycle 3: 634-637, 2004. 